\title{
First steps in development of a new transfer standard, for moisture measurement, based on radio-frequency wave and micro-wave
}

\author{
Georgin $\mathrm{E}^{1 \mathrm{a}}$, Rochas $\mathrm{JF}^{1}$, Hubert $\mathrm{S}^{1}$, Achard $^{1} \mathrm{P}$, Ben Ayoub $\mathrm{MW}^{1}$ \\ Sabouroux $\mathrm{P}^{2}$ \\ ${ }^{1}$ CETIAT, 25 avenue des arts - BP 52042, 69603 Villeurbanne, France \\ ${ }^{2}$ Institut Fresnel UMR CNRS 7249 - Aix-Marseille Universités Campus de Saint Jérôme, Avenue Escadrille Normandie-Niemen 13397 \\ Marseille Cedex
}

\begin{abstract}
Résumé. Le LNE-CETIAT fait partie d'un consortium, de laboratoires nationaux de métrologie, impliqué dans un projet européen de recherche en métrologie (Joint Research Project) dénommé SIB64 METefnet (www.metef.net) et financé par le comité European Metrology Research Programme (EMRP). L'objectif du projet est de permettre aux utilisateurs finaux de réaliser des mesures d'humidité dans les solides, fiables et traçables au SI, en améliorant les techniques de mesure et les méthodes d'étalonnage [1]. Parmi les différentes approches suivies par les partenaires de ce projet, le LNE-CETIAT a choisi de se concentrer sur la dissémination de la traçabilité au SI, à l'endroit d'utilisateurs industriels, en développement un instrument de transfert non destructif utilisant des Hautes-Fréquences et des micro-ondes, à bas niveau d'énergie $(0 \mathrm{dBm})$, pour mesurer l'humidité dans les matériaux solides.

La fréquence de relaxation de l'eau dans un matériau dépend, notamment, du degré de liaison entre les molécules d'eau et la matrice solide (substrat) [2-3]. La bande de fréquences couvre un spectre qui s'étend des HautesFréquences - HF, soit de quelques MHz à quelques centaines de $\mathrm{MHz}$, aux Micro-Ondes - MO, soit de quelques centaines de $\mathrm{MHz}$ à quelques $\mathrm{GHz}$. Ainsi, il est communément considéré, par les constructeurs utilisant les ondes électro-magnétiques - OEM comme moyen de chauffage dans les procédés de séchage, que l'eau liée physiquement ou chimiquement, à la matrice solide, interagit principalement et sélectivement avec les ondes du domaine RF, tandis que l'eau libre, dans la matrice solide, interagit principalement avec les MO (10 GHz).

L'instrument développé dans le cadre de ce projet exploite cette sélectivité, vis-à-vis du type d'OEM utilisée, en faisant des mesures de propriétés diélectriques des matériaux humides. Ce principe de mesure est connu et utilisé depuis les années 70, les instruments alors développés fonctionnant exclusivement soit en RF soit en MO et sur bande de fréquence extrêmement étroite. Ces appareils ne permettent donc pas de couvrir une large gamme de fréquence de sorte à optimiser la fréquence de mesure vis-à-vis du degré de liaison de l'eau avec la matrice solide.

L'originalité de ce travail repose sur la mise au point d'un instrument de mesure dont la fréquence de travail, utilisée pour mesurer l'humidité dans une matrice solide, est optimisée vis-à-vis de degré de liaison de l'eau avec le substrat et vis-à-vis de l'interaction entre l'OEM et le matériau. Pour ce faire, plus la fréquence de travail du capteur sera proche de la fréquence de relaxation mesurée plus la sensibilité de l'instrument sera bonne. Afin de déterminer la fréquence de relaxation de l'eau dans un solide, le CETIAT développe un équipement qui permet de couvrir une gamme de fréquences la plus grande possible. Pour ce besoin, deux cellules, non-résonnantes, ont été développées : une cellule capacitive et une cellule coaxiale. Cet article présente les cellules développées ainsi que les premiers résultats expérimentaux obtenus avec la cellule capacitive.
\end{abstract}

\section{Introduction}

\subsection{Framework of the project}

Formally, this work is a task included in a workpackage dedicated to "Traceability and dissemination". The aim of this workpackage is to develop improved methods for disseminating traceability from primary standards to industrial moisture measurements. Development of new transfer standards with traceability to the SI, among other possibilities such as the use of CRMs, plays a key role in reaching the aim.

Three National Metrology Institutes (NMI) or Designated Institutes (DI) are working on this task: INRIM (Italy), TUBITAK-UME (Turkey) and LNECETIAT (France). Two approaches are followed:

- Development of a non-invasive and nondestructive transfer standard based on radiofrequency wave and microwave at a low energy level for determination of moisture.

\footnotetext{
${ }^{a}$ Corresponding author: eric.georgin@cetiat.fr
} 
- Development of a low-cost, portable system comprising a microwave moisture meter and a system for maintaining an internal reference in very stable conditions.

LNE-CETIAT has chosen to focus on the first approach by developing a non-invasive and nondestructive new transfer standard based on radiofrequency $(\mathrm{RF})$ wave and micro-wave (MW) at low energy level for measuring moisture in materials.

\subsection{Principle}

The instrument, designed for this project, measures complex permittivity, $\varepsilon^{*}$, of a moist material by using radio-frequency wave and microwave at a low energy level. Permittivity is a quantity used to describe dielectric properties that influences reflection of electromagnetic (EM) waves at interfaces and the attenuation of wave energy within materials. In frequency domain, $\varepsilon^{*}$ of a material to that of free space can be expressed as:

$$
\begin{gathered}
\varepsilon^{*}=\varepsilon^{\prime}-j \varepsilon^{\prime \prime}=\varepsilon_{0}\left(\varepsilon^{\prime}-j \varepsilon^{\prime \prime}\right)=\varepsilon^{\prime}-j \varepsilon^{\prime} \tan \delta \\
\tan \delta=\frac{\varepsilon^{\prime \prime}}{\varepsilon^{\prime}}=\frac{\varepsilon_{r}^{\prime \prime}}{\varepsilon_{r}^{\prime}} \\
\varepsilon_{r}^{\prime}=\frac{\varepsilon^{\prime}}{\varepsilon_{0}} \text { and } \varepsilon_{r}^{\prime \prime}=\frac{\varepsilon^{\prime \prime}}{\varepsilon_{0}}
\end{gathered}
$$

$j=\sqrt{-1}, \tan \delta$ is the tangent of loss angle, the real part $\varepsilon^{\prime}$ is referred to as the dielectric constant and represents stored energy when the material is exposed to an electric field, while the imaginary part $\varepsilon^{\prime \prime}$ is referred to the dielectric loss factor and influences energy absorption and attenuation; see Figure 1. $\varepsilon_{r}^{\prime}$ and $\varepsilon_{r}^{\prime \prime}$ are relative permittivity with respect to permittivity of the vaccum $\varepsilon_{0}$.

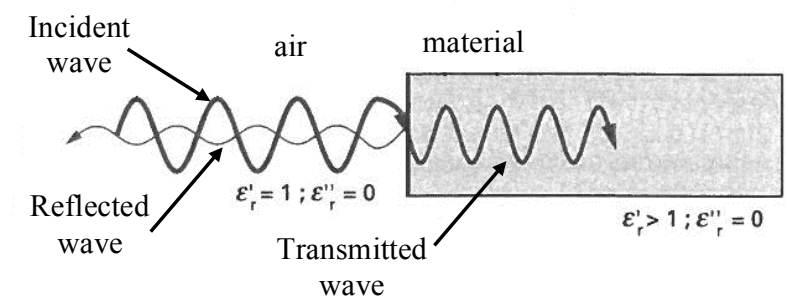

Incident wave propagating from air to a material without losses

$$
\left(\varepsilon^{\prime \prime}=0\right)
$$

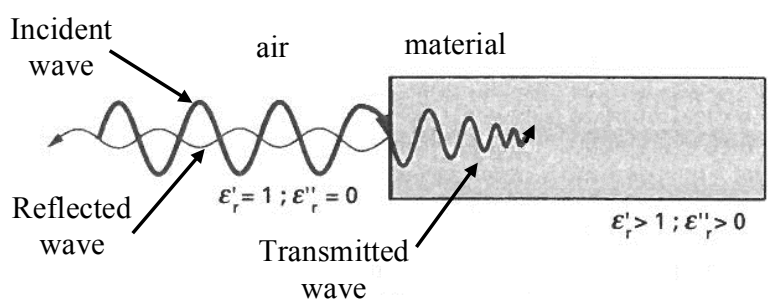

Incident wave propagating from air to a material with losses

$$
\left(\varepsilon^{\prime \prime}>0\right)
$$

Figure 1 Wave propagation between two media [6]

Thus the power absorbed by a material due to an EM wave can be expressed as:

$$
P_{\text {absorb }}=2 \pi \varepsilon_{0} E_{a}^{2} \varepsilon^{\prime} \tan \delta V
$$

Dielectric properties of materials are affected by many factors, including frequency, temperature, and moisture content. $\varepsilon^{\prime}$ and $\varepsilon^{\prime \prime}$ can also vary significantly with frequency, and, for example in case of a mist material, the frequency dependence of dielectric properties can provide information about the degree of binding and volume of the water inside the material.

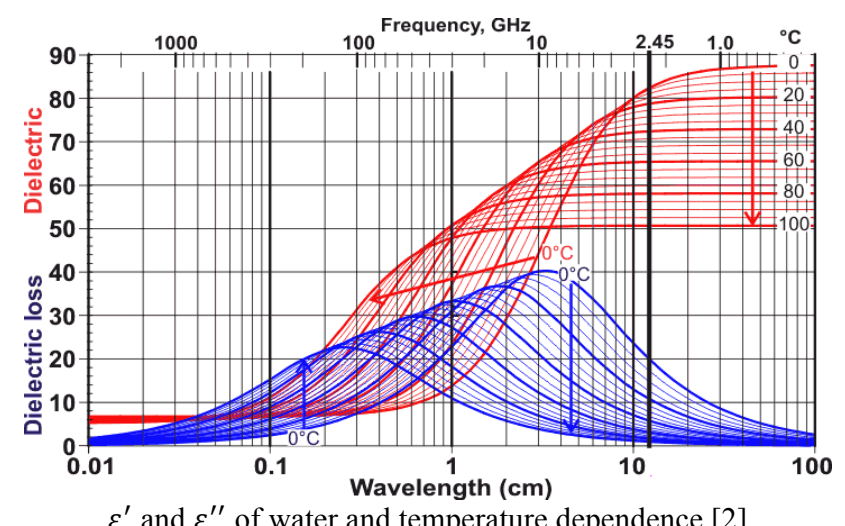

$\varepsilon^{\prime}$ and $\varepsilon^{\prime \prime}$ of water and temperature dependence [2]

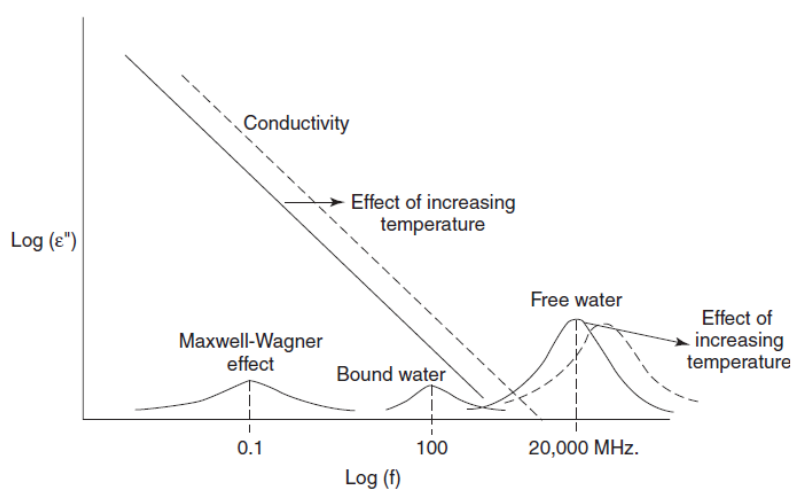

Contributions of various mechanisms $\varepsilon^{\prime \prime}$ for moist materials [4]

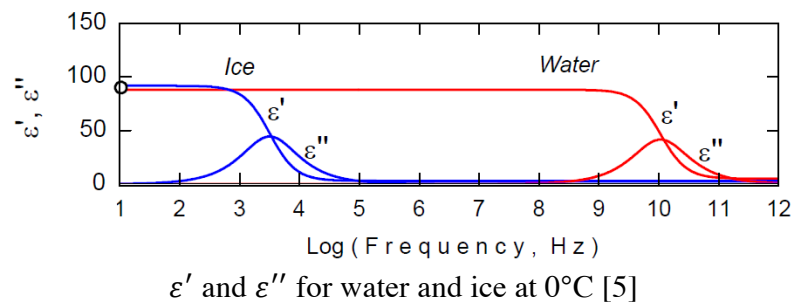

Figure 2 dielectric permittivity, $\boldsymbol{\varepsilon}^{\prime}$ and $\boldsymbol{\varepsilon}^{\prime \prime}$, and influence quantities

As it has been shown just above, the relaxation frequency of the water in a material depends on the degree of binding of the water with the material (substrat) with which it coexists.

The domain of these frequencies, investigated in this project, covers a spectrum which extends from RF, i.e. from few $\mathrm{MHz}$ to some hundreds of $\mathrm{MHz}$, to $\mathrm{MW}$, i.e. from few hundreds of MHZ to some tens of GHz. It is commonly admitted, in particular by manufacturers which use the EM waves as means of heating in drying process, that bounded water is mainly and selectively excited by RF waves while free water is more excited by microwaves. This selectivity clearly appears while measuring dielectric impedance properties and this specific behaviour is the key point that the instrument will use in the project. 
The measurement principle is known since the seventies leading to development of instrument working exclusively in RF or in microwaves frequencies. In addition the frequency bandwidth of these instruments is very narrow, which did not allow to scan a large bandwidth and thus to optimize the frequency measurement according to the degree of binding and the type of material.

The innovative aspect rely on the fact that the best the working frequency to measure dielectric impedance properties, and thus the moisture, is imposed by the degree of binding and the interaction with the material. The closer the measurement frequency is to the relaxation frequency the better the sensitivity is. To determine the water relaxation frequency in a solid, CETIAT develops equipment that can cover a range of the maximum possible frequencies. For this need, two family cells, nonresonant, have been developed: a capacitive cell and a coaxial cells.

\section{Experimental set up}

The instrument is mainly composed by a Virtual Network Analyser (VNA) MS2038C - $5 \mathrm{kHz}$ to $20 \mathrm{GHz}$, and 2 kind of experimental cells: capacitive and coaxial. All these cells are non-resonant and cover a broad band of frequencies.

\subsection{Capacitive cell}

This cell covers the frequency range from $1 \mathrm{MHz}$ to $400 \mathrm{MHz}$. Two sets of electrodes have been designed: one for solid materials and one for liquids.
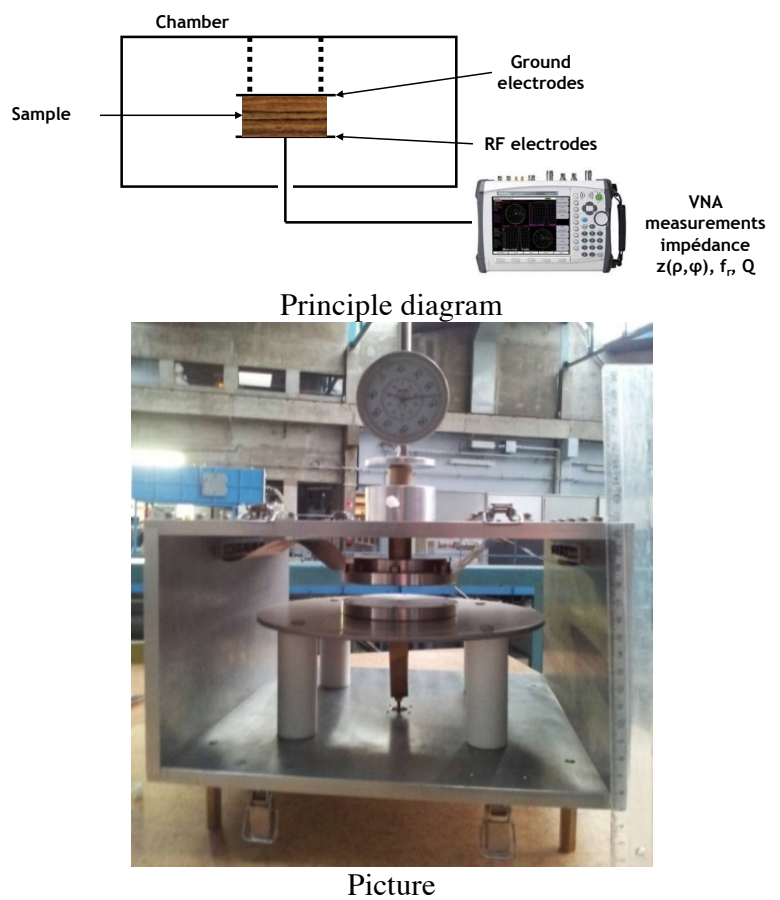

Figure 3 Capacitive cell

The experimental cell is composed by chamber which housed two electrodes, see Figure 3 . The moist material to be analysed is placed between electrodes and stands for a dielectric to be measured.
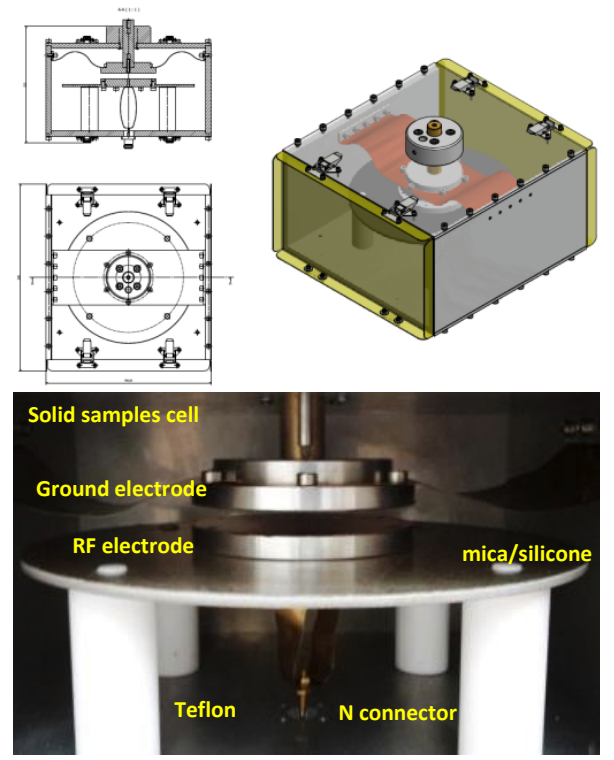

Capacitive cell for solid materials
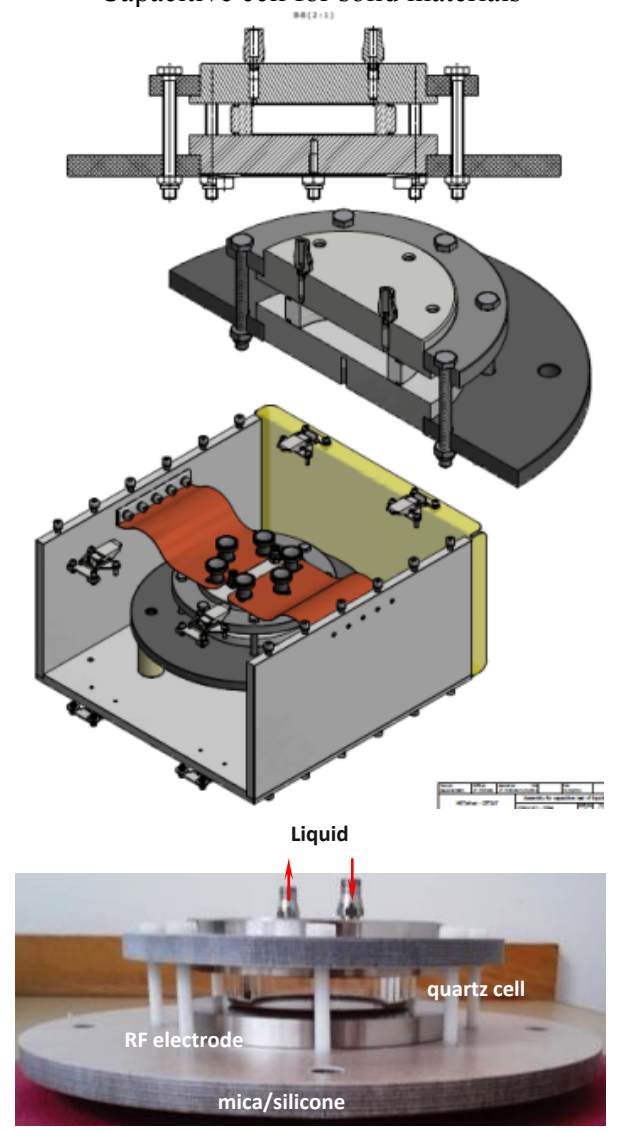

Capacitive cell for liquid

Figure 4 Technical drawings and pictures of experimental cells designed

Impedance measurements lead to complex permittivity determination. 


\subsection{Coaxial cell}

\subsubsection{Cell developed at LNE-CETIAT}

This cell covers the frequency range from $200 \mathrm{MHz}$ to $4 \mathrm{GHz}$. Two sets of electrodes have been designed: one for solid materials and one for liquids.

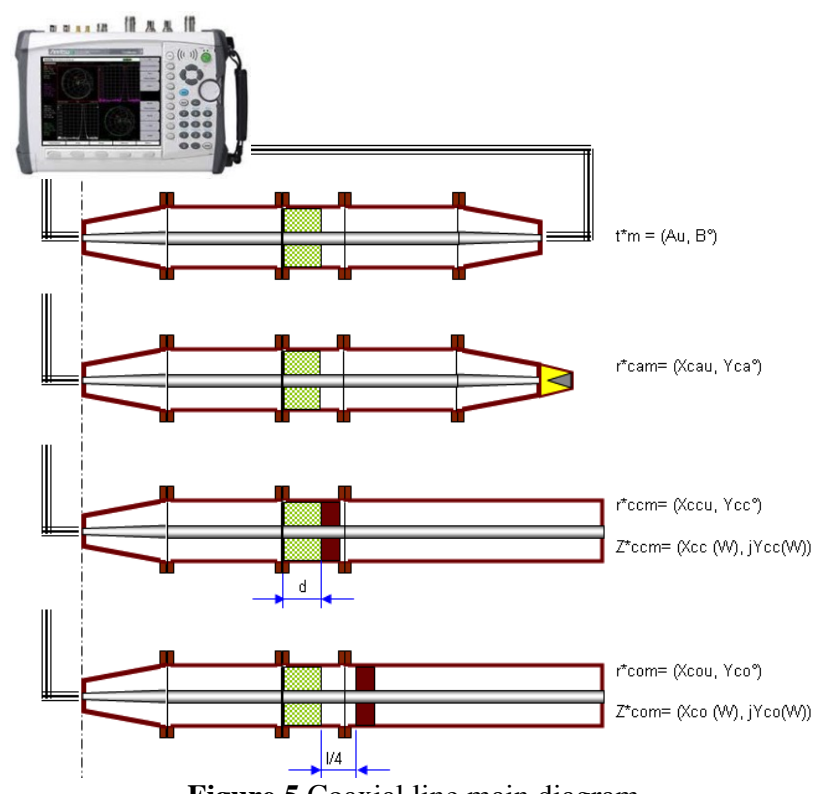

Figure 5 Coaxial line main diagram

This cell is very close to a wave guide and allows four measurements configurations (transmission, matched load, short circuit and open circuit). It leads to a set of 8 equations for determining complex permittivity.
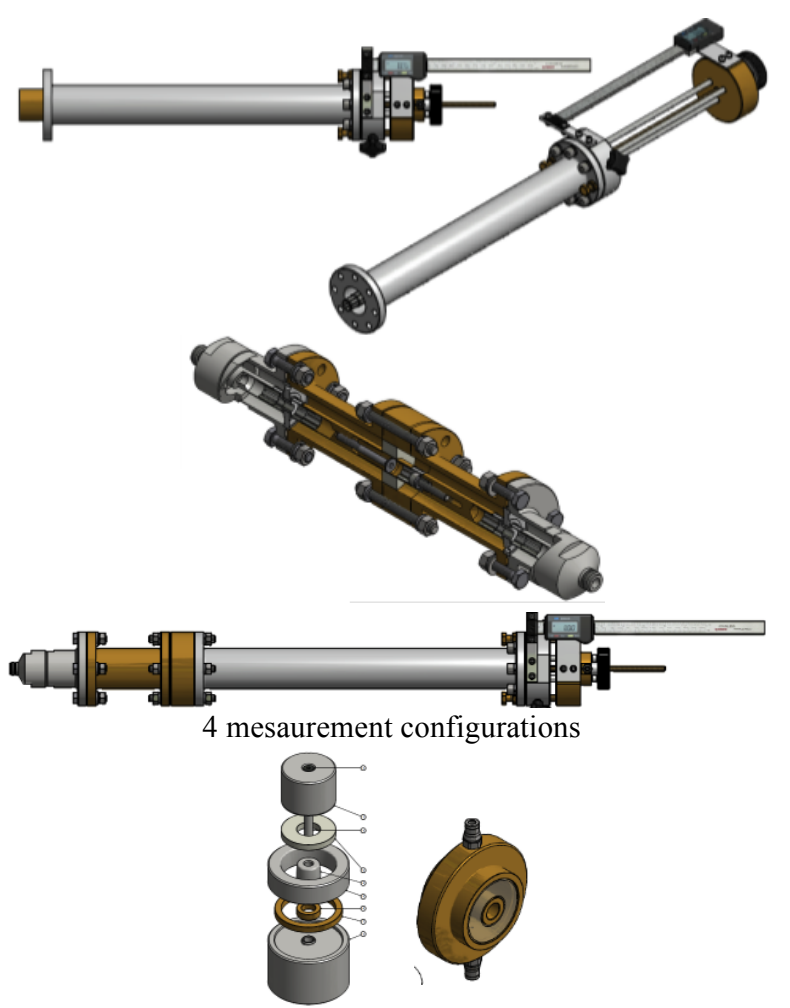

Sample holders: solid material (left) liquid (right)

Figure 6 Technical drawings of coaxial line
During the qualification process of experimental set up, it is scheduled to organize a comparison with a tool, EpsiMu, that have been purchased thanks to a new collaboration with Fresnel Institute

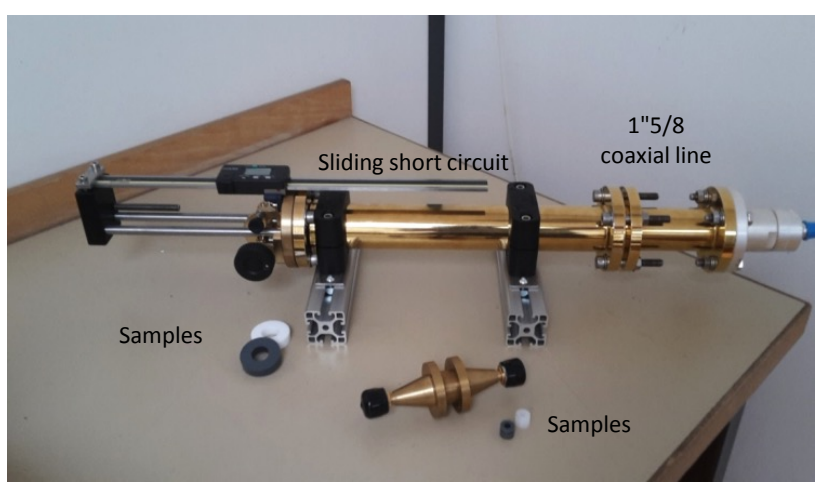

Figure 7 Coaxial cell and Epsimu cell

\subsubsection{Cell developed at Fresnel Institute}

Fresnel Institute has developed EpsiMu (http://www.epsimu.fr/); it is a tool designed for dielectric properties measurement. This tool can be used to characterize all types of materials. It consists of a coaxial cell and a dedicated software that allows to reconstruct the permittivity in almost real-time by a deembedding process [7-9].

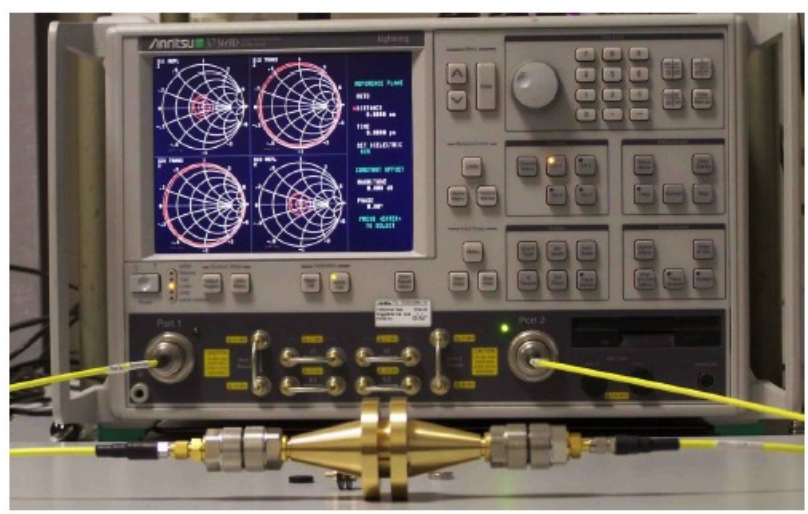

Figure 8 Ерsimu tool for dielectric properties measurement

\subsection{Complementary means}

On traceability point of view, moisture content measurement technics can be defined in two categories:

- Direct measurement: the property of interest is directly measured according to SI primary unit. It concerns reference or absolute moisture content measurement techniques such as:

- (Thermo)gravimetric methods (Loss on drying method, gain on wetting, thermogravimetric analysis)

- Karl Fischer titration (Volumetric, Coulometric)

- Indirect measurement: it is measured by another property and linked to the property of interest through a calibration. It concerns secondary or inferential moisture content measurement techniques such as: 
- Electromagnetic techniques (Time Domain Reflectometry (TDR), electrical capacitance, dielectric permittivity/spectroscopy, micro waves and radio frequencies absorption, ...)

- Thermal techniques (Infrared (IR) retrodiffusion / Near Infrared (NIR) spectroscopy,...)

- Nuclear techniques (Nuclear Magnetic Resonance (NMR), Neutron moderation,...)

In order to ensure traceability to SI of the experimental set up developed in the project, the laboratory is equipped with an infrared moisture analyzer MA100C, with a weighing capacity of $100 \mathrm{~g}$ with $0.1-\mathrm{mg}$ weight resolution.

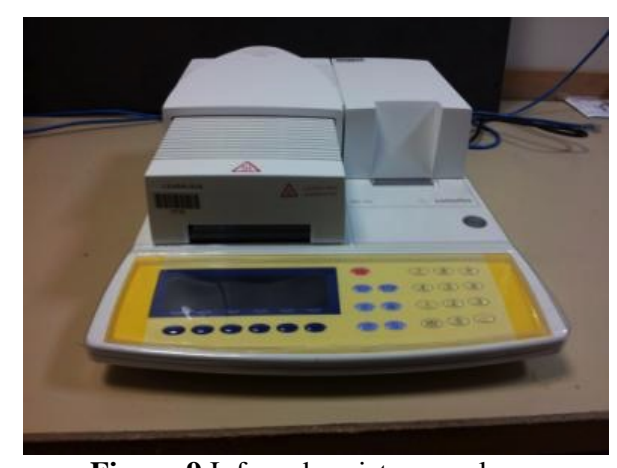

Figure 9 Infrared moisture analyzer

For measuring the temperature in the EM field, it is necessary to use a probe which interacts, the less as possible, with the EM field. Thus we use optical fiber sensor for the temperature measurement.

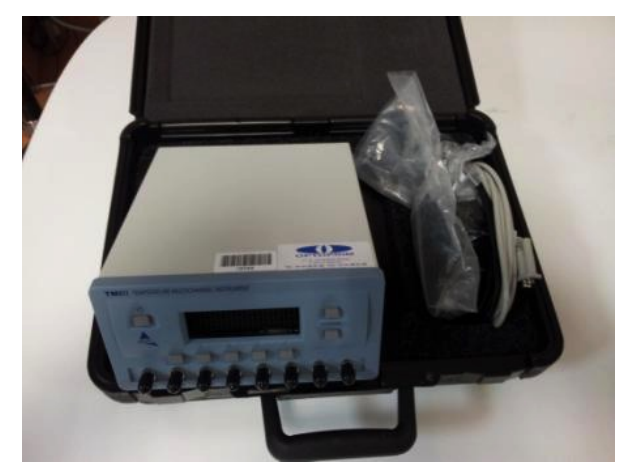

TMI 8-channel temperature fiber optic signal conditioner

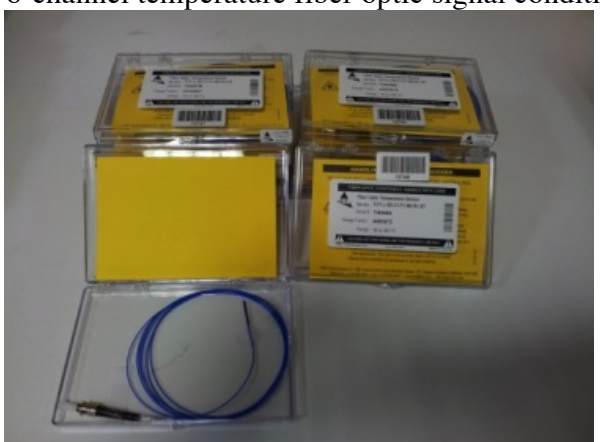

Fiber optic temperature sensor; range of $-40{ }^{\circ} \mathrm{C}$ to $300{ }^{\circ} \mathrm{C}$, diameter $50 \mu \mathrm{m}$

Figure 10 Temperature measuring chain adapted for EM field

\section{First experimental results}

Before measuring moist materials, the experimental cells need to be characterized in terms of EM behaviour. In this work we have, first, begun with the capacitive cell.

The key point is to be sure to handle correctly the electrical behaviour of the cell. Indeed, thanks to this, the determination of $\varepsilon^{\prime}$ and $\varepsilon^{\prime \prime}$, from the measurements performed, will be correct. For this purpose it is convenient to study a reference materials well described on dielectric point of view. In the literature, liquid materials are often well studied in a broad band of frequencies, which is not the case for solid materials.

Thus, the characterization of capacitive cell has been carried out with two "liquid type" cells: $\varnothing 60 \mathrm{~mm}$ and $\varnothing$ $80 \mathrm{~mm}$. First, the electrical behaviour and equations used are presented. Then the results obtained for two fluids, Heptanol $\left(\mathrm{C}_{7} \mathrm{H}_{15} \mathrm{OH}\right)$ and Cyclohexanol $\left(\mathrm{C}_{6} \mathrm{H}_{11} \mathrm{OH}\right)$, are presented hereafter and compared to reference values [10]

\subsection{Computation of permittivity for the capacitive cell composed by two parallel circular electrodes}

\subsubsection{Measurement without material}

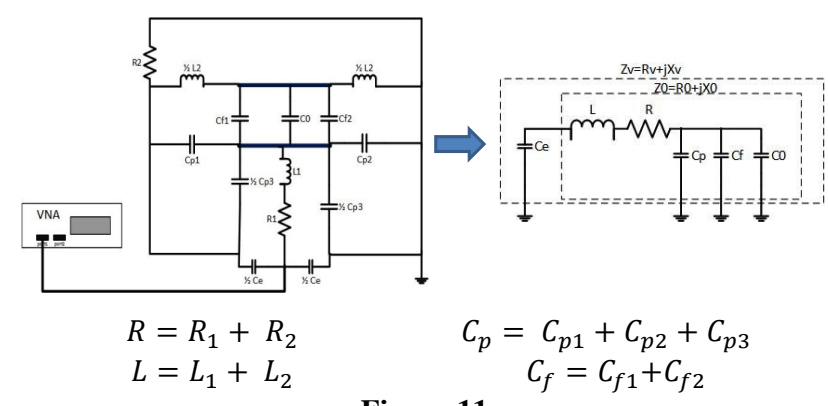

Figure 11

1. Expression of $\mathrm{ZO}$ from the impedance measured Zv with VNA

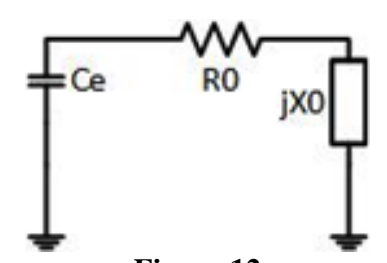

Figure 12

$$
\begin{aligned}
\frac{1}{Z v} & =\frac{1}{R v+j X v}=j C e \omega+\frac{1}{R 0+j X 0} \\
R 0+j X 0 & =\frac{1}{\frac{1}{Z v}-j C e \omega}=\frac{(R v+j X v)(X v C e \omega+1)+j(C e \omega R v)}{(X v C e \omega+1)^{2}+(\operatorname{Ce} \omega R)^{2}}
\end{aligned}
$$

Identification leads to:

$$
\begin{gathered}
R 0=\frac{R v}{(X v C e \omega+1)^{2}+(C e \omega R v)^{2}} \\
X 0=\frac{(X v C e \omega+1) X v+R v(C e \omega R v)}{(X v C e \omega+1)^{2}+(C e \omega R v)^{2}}
\end{gathered}
$$


2. Parasitic capacities $\mathrm{Cp}$ and $\mathrm{Cf}$ as a function of $\mathrm{RO}$ and $\mathrm{XO}$

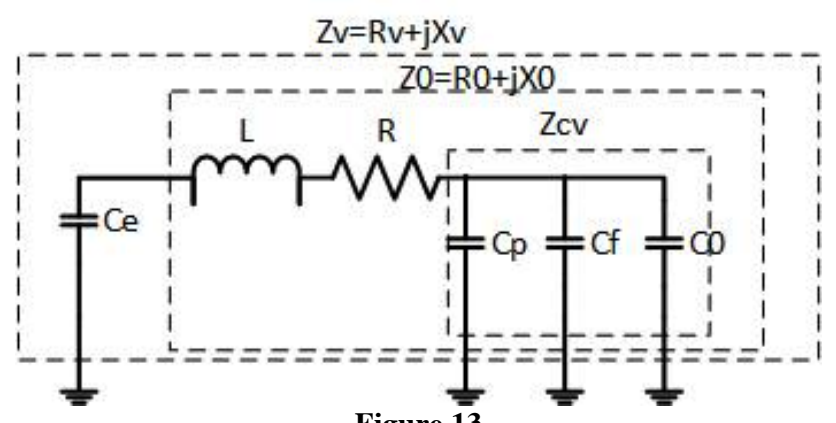

Figure 13

$$
\begin{gathered}
\frac{1}{z_{c v}}=\frac{1}{\frac{1}{j\left(C_{p}+C_{f}+C_{0}\right) \omega}}=j \omega\left(C_{p}+C_{f}\right)+j \omega C_{0} \\
=j \omega\left(C_{\text {Totale }}\right)+j \omega C_{0}
\end{gathered}
$$

With $\mathrm{C}_{0}=\frac{\varepsilon_{0} \varepsilon_{\mathrm{a}}^{\prime} \mathrm{A}_{\mathrm{p}}}{\mathrm{d}}$ and $\mathrm{C}_{\text {Totale }}=\mathrm{C}_{\mathrm{p}}+\mathrm{C}_{\mathrm{f}}$

$$
Z_{c v}=-j \frac{1}{C_{\text {Totale }} \omega+C_{0} \omega}
$$

It comes $\mathrm{Z}_{0}$ :

$$
Z_{0}=R+j L \omega+Z_{c v}=R+j\left(L \omega-\frac{1}{C_{\text {Totale }} \omega+C_{0} \omega}\right)
$$

From equation 6 it comes:

$$
Z_{0}=R_{0}+j X_{0}
$$

Identification leads to:

$$
R_{0}=R
$$

$$
\begin{gathered}
X_{0}=L \omega-\frac{1}{\omega\left(C_{\text {Totale }}+C_{0}\right)}=\frac{L \omega^{2}\left(C_{\text {Totale }}+C_{0}\right)-1}{\omega\left(C_{\text {Totale }}+C_{0}\right)} \\
=\frac{L C_{\text {Totale }} \omega^{2}+L C_{0} \omega^{2}-1}{\omega\left(C_{\text {Totale }}+C_{0}\right)} \\
X_{0} \omega\left(C_{\text {Totale }}+C_{0}\right)=L C_{\text {Totale }} \omega^{2}+L C_{0} \omega^{2}-1 \\
X_{0} \omega C_{\text {Totale }}+X_{0} \omega C_{0}=L C_{\text {Totale }} \omega^{2}+L C_{0} \omega^{2}-1 \\
X_{0} \omega C_{\text {Totale }}-L C_{\text {Totale }} \omega^{2}=L C_{0} \omega^{2}-X_{0} C_{0} \omega-1 \\
C_{\text {Totale }}\left(X_{0} \omega-L \omega^{2}\right)=L C_{0} \omega^{2}-X_{0} C_{0} \omega-1
\end{gathered}
$$

Finally:

$$
\begin{aligned}
C_{\text {Totale }}=\frac{L C_{0} \omega^{2}-X_{0} C_{0} \omega-1}{\omega\left(X_{0}-L \omega\right)} & \\
= & -\frac{\varepsilon_{0} \varepsilon_{a}^{\prime} A_{p}}{d}-\frac{1}{\omega\left(X_{0}-L \omega\right)}
\end{aligned}
$$

\subsubsection{Measurement with material (sample)}

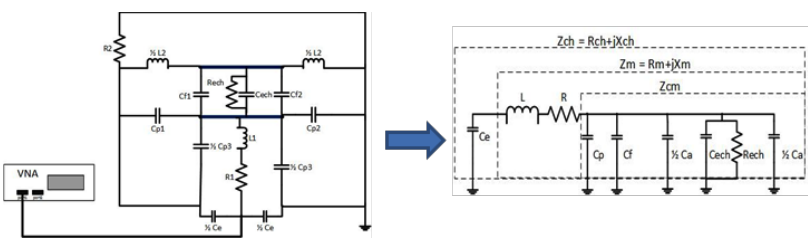

Figure 14

1. Expression of $\mathrm{Zm}$ as a function of $\mathrm{Zch}$ measured with VNA

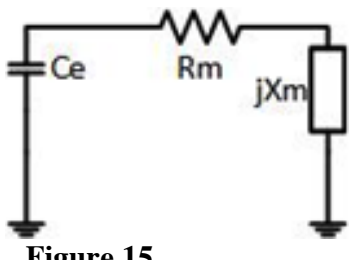

Figure 15

$$
\begin{gathered}
\frac{1}{\mathrm{Zch}}=\frac{1}{\mathrm{Rch}+\mathrm{jXch}}=\mathrm{j} \text { Ce } \omega+\frac{1}{\mathrm{Rm}+\mathrm{jXm}} \\
\mathrm{Rm}+\mathrm{jXm}=\frac{1}{\frac{1}{\mathrm{Zch}}-\mathrm{jCe} \omega} \\
=\frac{(\mathrm{Rch}+\mathrm{jXch})(\mathrm{Xch} \cdot \mathrm{Ce} \cdot \omega+1)+\mathrm{j}(\mathrm{Ce} \cdot \omega \cdot \mathrm{Rch})}{\left((\text { Xch. Ce. } \omega+1)^{\wedge} 2+(\text { Ce. } \omega \cdot \text { Rch })^{\wedge} 2\right)}
\end{gathered}
$$

Identification leads to

$$
\begin{gathered}
\mathrm{Rm}=\frac{\mathrm{Rch}}{(\text { Xch. Ce. } \omega+1)^{2}+(\text { Ce. } \omega . \text { Rch })^{2}} \\
\mathrm{Xm}=\frac{(\text { Xch. Ce. } \omega+1) \text { Xch }+ \text { Rch }(\text { Ce } \omega \cdot \text { Rch })}{(\text { Xch } . \text { Ce. } \omega+1)^{2}+(\text { Ce. } \omega . \text { Rch })^{2}}
\end{gathered}
$$

\section{Expression of Cech and Rech as function of material characteristics}

Nota Bene: in this development, sample diameter should be always lower than electrode diameter

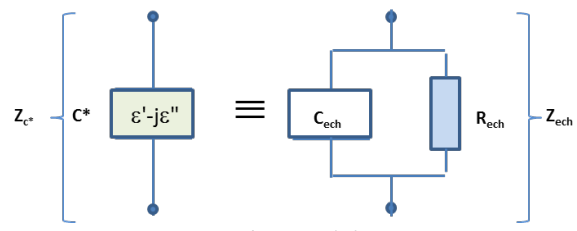

Figure 16

$$
\begin{array}{r}
\frac{1}{Z_{e c h}}=\frac{1}{R_{e c h}}+\frac{1}{\frac{1}{j \omega C_{e c h}}}=\frac{1}{R_{e c h}}+j \omega C_{e c h} \\
=\frac{1+j \omega C_{e c h} R_{e c h}}{R_{e c h}} \\
Z_{e c h}=\frac{R_{e c h}\left(1-j \omega C_{e c h} R_{e c h}\right)}{\left(1+j \omega C_{e c h} R_{e c h}\right)\left(1-j \omega C_{e c h} R_{e c h}\right)} \\
=\frac{R_{e c h}}{1+\omega^{2} C_{e c h}^{2} R_{e c h}^{2}} \\
-j \frac{\omega C_{e c h} R_{e c h}^{2}}{1+\omega^{2} C_{e c h}^{2} R_{e c h}^{2}}
\end{array}
$$

And 


$$
\begin{gathered}
Z_{C *}=\frac{1}{j \omega C^{*}}=\frac{1}{j \omega \frac{\varepsilon_{0}\left(\varepsilon^{\prime}-j \varepsilon^{\prime \prime}\right) A_{m}}{d}}=\frac{d}{j \omega \varepsilon_{0} A_{m}\left(\varepsilon^{\prime}-j \varepsilon^{\prime \prime}\right)} \\
=\frac{d}{\omega \varepsilon_{0} A_{m}\left(\varepsilon^{\prime \prime}+j \varepsilon^{\prime}\right)}
\end{gathered}
$$

With $A_{m}$ sample surface and $d$ sample diameter.

$$
\begin{aligned}
Z_{C *}=\frac{d\left(\varepsilon^{\prime \prime}-j \varepsilon^{\prime}\right)}{\omega \varepsilon_{0} A_{m}\left(\varepsilon^{\prime 2}+\varepsilon^{\prime \prime 2}\right)} \\
=\frac{d \varepsilon^{\prime \prime}}{\omega \varepsilon_{0} A_{m}\left(\varepsilon^{\prime 2}+\varepsilon^{\prime \prime 2}\right)} \\
-j \frac{d \varepsilon^{\prime}}{\omega \varepsilon_{0} A_{m}\left(\varepsilon^{\prime 2}+\varepsilon^{\prime \prime 2}\right)}
\end{aligned}
$$

With an identification of real and imaginary part in equation 25 and equation 27, it comes:

$$
\begin{aligned}
& \frac{R_{e c h}}{1+\omega^{2} C_{e c h}^{2} R_{e c h}^{2}}=\frac{d \varepsilon^{\prime \prime}}{\omega \varepsilon_{0} A_{m}\left(\varepsilon^{\prime 2}+\varepsilon^{\prime \prime 2}\right)} \\
& \frac{\omega C_{e c h} R_{e c h}^{2}}{1+\omega^{2} C_{e c h}^{2} R_{e c h}^{2}}=\frac{d \varepsilon^{\prime}}{\omega \varepsilon_{0} A_{m}\left(\varepsilon^{\prime 2}+\varepsilon^{\prime \prime 2}\right)}
\end{aligned}
$$

By dividing equation 28 and equation 29, it comes:

$$
\begin{aligned}
\frac{1}{\omega C_{e c h} R_{e c h}}=\frac{\varepsilon^{\prime \prime}}{\varepsilon^{\prime}} \rightarrow & \varepsilon^{\prime}=\omega C_{e c h} R_{e c h} \varepsilon^{\prime \prime} \rightarrow R_{e c h}=\frac{\varepsilon^{\prime}}{\omega C_{e c h} \varepsilon^{\prime \prime}} \\
= & \frac{1}{\omega C_{e c h} \tan \delta}
\end{aligned}
$$

By replacing Rech in equation 28 :

$$
\begin{aligned}
& \frac{\frac{1}{\omega C_{e c h} \tan \delta}}{1+\omega^{2} C_{e c h}^{2}\left(\frac{1}{\omega C_{e c h} \tan \delta}\right)^{2}}=\frac{d \varepsilon^{\prime \prime}}{\omega \varepsilon_{0} A_{m}\left(\varepsilon^{\prime 2}+\varepsilon^{\prime \prime}\right)} \\
& =\frac{d \varepsilon^{\prime} \tan \delta}{\omega \varepsilon_{0} A_{m} \varepsilon^{\prime 2}\left(1+\tan \delta^{2}\right)} \\
& =\frac{d \tan \delta}{\omega \varepsilon_{0} A_{m} \varepsilon^{\prime}\left(1+\tan \delta^{2}\right)}
\end{aligned}
$$

Thus Cech and Rech expressed as a function of dielectric parameters of the material or sample:

$$
\begin{gathered}
C_{e c h}=\frac{\varepsilon_{0} \varepsilon^{\prime} A_{m}}{d} \\
R_{e c h}=\frac{d}{\omega \varepsilon_{0} \varepsilon^{\prime \prime} A_{m}}=\frac{d}{\omega \varepsilon_{0} A_{m} \varepsilon^{\prime} \tan \delta}=\frac{1}{\omega C_{e c h} \tan \delta}
\end{gathered}
$$

\section{Expression of $\mathrm{Zcm}$ as a fuction of $\varepsilon^{\prime}$ and} $\varepsilon^{\prime \prime}$

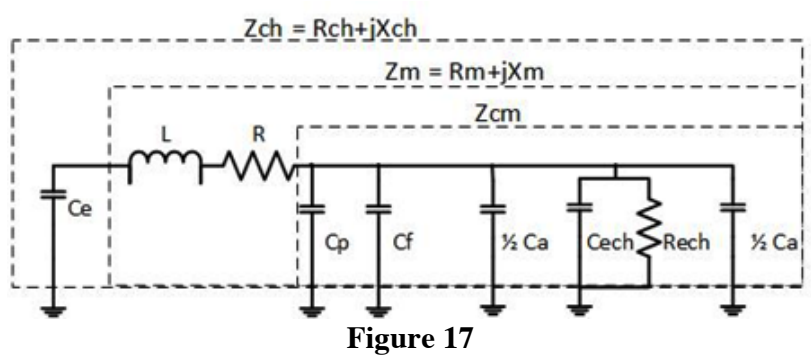

$$
\frac{1}{Z_{c m}}=\frac{1}{R_{e c h}}+j \omega\left(C_{p}+C_{f}+C_{a}+C_{e c h}\right)
$$

With

$$
C_{a}=\frac{\varepsilon_{0} \varepsilon_{a}^{\prime}\left(A_{p}-A_{m}\right)}{d}
$$

$$
\begin{gathered}
C_{\text {Totale }}=C_{p}+C_{f}=\frac{L C_{0} \omega^{2}-X_{0} C_{0} \omega-1}{\omega\left(X_{0}-L \omega\right)} \\
=\frac{1}{\omega\left(L \omega-X_{0}\right)}-\frac{\varepsilon_{0} \varepsilon_{a}^{\prime} A_{p}}{d} \\
C_{\text {Totale }}+C_{a}=\frac{1}{\omega\left(L \omega-X_{0}\right)}-\frac{\varepsilon_{0} \varepsilon_{a}^{\prime} A_{p}}{d}+\frac{\varepsilon_{0} \varepsilon_{a}^{\prime} A_{p}}{d} \\
-\frac{\varepsilon_{0} \varepsilon_{a}^{\prime} A_{m}}{d}=\frac{1}{\omega\left(L \omega-X_{0}\right)}-\frac{\varepsilon_{0} \varepsilon_{a}^{\prime} A_{m}}{d} \\
C_{\text {Totale }}+C_{a}+C_{e c h}=\frac{1}{\omega\left(L \omega-X_{0}\right)}-\frac{\varepsilon_{0} \varepsilon_{a}^{\prime} A_{m}}{d}+\frac{\varepsilon_{0} \varepsilon^{\prime} A_{m}}{d} \\
\frac{1}{Z_{c m}}=\frac{\omega \varepsilon_{0} A_{m} \varepsilon^{\prime} \tan \delta}{d} \quad \begin{array}{c}
1 \\
+j \omega\left(\frac{\varepsilon_{0} \varepsilon_{a}^{\prime} A_{m}}{d}\right.
\end{array} \\
\left.+\frac{\varepsilon_{0} \varepsilon^{\prime} A_{m}}{d}\right)
\end{gathered}
$$

By replacing $\frac{1}{\omega\left(L \omega-X_{0}\right)}-\frac{\varepsilon_{0} \varepsilon_{a}^{\prime} A_{m}}{d}$ by $B$

$$
\begin{gathered}
\frac{1}{Z_{c m}}=\frac{\omega \varepsilon_{0} A_{m} \varepsilon^{\prime} \tan \delta}{d}+j \omega\left(B+\frac{\varepsilon_{0} \varepsilon^{\prime} A_{m}}{d}\right) \\
Z_{c m}=\frac{d}{\omega \varepsilon_{0} A_{m} \varepsilon^{\prime} \tan \delta+j \omega\left(B d+\varepsilon_{0} \varepsilon^{\prime} A_{m}\right)} \\
Z_{c m}=\frac{d\left[\omega \varepsilon_{0} A_{m} \varepsilon^{\prime} \tan \delta-j \omega\left(B d+\varepsilon_{0} \varepsilon^{\prime} A_{m}\right)\right]}{\left(\omega \varepsilon_{0} A_{m} \varepsilon^{\prime} \tan \delta\right)^{2}+\left(\omega\left(B d+\varepsilon_{0} \varepsilon^{\prime} A_{m}\right)\right)^{2}}
\end{gathered}
$$

\section{Determination of $\varepsilon^{\prime}$ and $\varepsilon^{\prime \prime}$ from $\mathrm{Rm}$ and Xm}

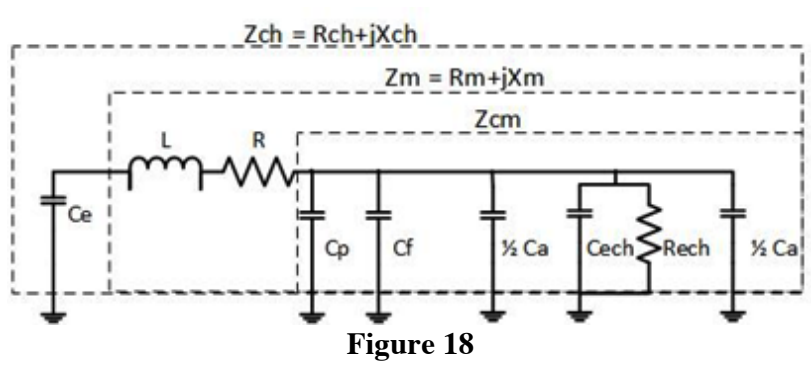




$$
Z_{m}=R_{m}+j X_{m}
$$

$\mathrm{Rm}$ and Xm have been calculated from Rch and Xch, measured quantities with VNA, when electrodes contain a material or sample

$$
\begin{gathered}
R_{m}=R+\frac{d \omega \varepsilon_{0} A_{m} \varepsilon^{\prime} \tan \delta}{\left(\omega \varepsilon_{0} A_{m} \varepsilon^{\prime} \tan \delta\right)^{2}+\left[\omega\left(B d+\varepsilon_{0} \varepsilon^{\prime} A_{m}\right)\right]^{2}} \\
X_{m}=L \omega-\frac{d \omega\left(B d+\varepsilon_{0} \varepsilon^{\prime} A_{m}\right)}{\left(\omega \varepsilon_{0} A_{m} \varepsilon^{\prime} \tan \delta\right)^{2}+\left[\omega\left(B d+\varepsilon_{0} \varepsilon^{\prime} A_{m}\right)\right]^{2}} \\
R_{m}-R=\frac{d \omega \varepsilon_{0} A_{m} \varepsilon^{\prime} \tan \delta}{\left(\omega \varepsilon_{0} A_{m} \varepsilon^{\prime} \tan \delta\right)^{2}+\left[\omega\left(B d+\varepsilon_{0} \varepsilon^{\prime} A_{m}\right)\right]^{2}} \\
X_{m}-L \omega=-\frac{d \omega\left(B d+\varepsilon_{0} \varepsilon^{\prime} A_{m}\right)}{\left(\omega \varepsilon_{0} A_{m} \varepsilon^{\prime} \tan \delta\right)^{2}+\left[\omega\left(B d+\varepsilon_{0} \varepsilon^{\prime} A_{m}\right)\right]^{2}}
\end{gathered}
$$

With $\quad R_{m}-R_{l}=C$ and $\quad X_{m}-L \omega=D$

$$
\begin{aligned}
& \frac{D}{C}=-\frac{\left(B d+\varepsilon_{0} \varepsilon^{\prime} A_{m}\right)}{\varepsilon_{0} A_{m} \varepsilon^{\prime} \tan \delta} \\
& D \varepsilon_{0} A_{m} \varepsilon^{\prime} \tan \delta=-C\left(B d+\varepsilon_{0} \varepsilon^{\prime} A_{m}\right) \\
& D \varepsilon_{0} A_{m} \varepsilon^{\prime} \tan \delta=-C B d-C \varepsilon_{0} \varepsilon^{\prime} A_{m} \\
& D \varepsilon_{0} A_{m} \varepsilon^{\prime \prime}+C \varepsilon_{0} \varepsilon^{\prime} A_{m}=-C B d \\
& C \varepsilon_{0} \varepsilon^{\prime} A_{m}=-D \varepsilon_{0} A_{m} \varepsilon^{\prime \prime}-C B d \\
& \varepsilon^{\prime}=\frac{-D \varepsilon_{0} A_{m} \varepsilon^{\prime \prime}-C B d}{C \varepsilon_{0} A_{m}}=-\frac{D \varepsilon}{C}-\frac{B d}{\varepsilon_{0} A_{m}} \\
& C=\frac{d \omega \varepsilon_{0} A_{m} \varepsilon^{\prime} \tan \delta}{\left(\omega \varepsilon_{0} A_{m} \varepsilon^{\prime} \tan \delta\right)^{2}+\left[\omega\left(B d+\varepsilon_{0} \varepsilon^{\prime} A_{m}\right)\right]^{2}} \\
& C=\frac{d \omega \varepsilon_{0} A_{m} \varepsilon^{\prime \prime}}{\left(\omega \varepsilon_{0} A_{m} \varepsilon^{\prime \prime}\right)^{2}+\left[\omega\left(B d+\varepsilon_{0} \varepsilon^{\prime} A_{m}\right)\right]^{2}} \\
& C\left\{\left(\omega \varepsilon_{0} A_{m} \varepsilon^{\prime \prime}\right)^{2}+\left[\omega\left(B d+\varepsilon_{0} \varepsilon^{\prime} A_{m}\right)\right]^{2}\right\}=d \omega \varepsilon_{0} A_{m} \varepsilon^{\prime \prime} \\
& C\left(\omega \varepsilon_{0} A_{m} \varepsilon^{\prime \prime}\right)^{2}+C \omega^{2}\left[B d+\varepsilon_{0}\left(-\frac{D \varepsilon "}{C}-\frac{B d}{\varepsilon_{0} A_{m}}\right) A_{m}\right]^{2} \\
& =d \omega \varepsilon_{0} A_{m} \varepsilon^{\prime \prime} \\
& C\left(\omega \varepsilon_{0} A_{m} \varepsilon^{\prime \prime}\right)^{2}+C \omega^{2}\left[B d-\frac{\varepsilon_{0} D \varepsilon^{\prime \prime} A_{m}}{C}-\frac{\varepsilon_{0} B d A_{m}}{\varepsilon_{0} A_{m}}\right]^{2} \\
& =d \omega \varepsilon_{0} A_{m} \varepsilon^{\prime \prime} \\
& C \omega^{2} \varepsilon_{0}{ }^{2} A_{m}{ }^{2} \varepsilon^{\prime \prime 2}+C \omega^{2}\left(-\frac{\varepsilon_{0} D \varepsilon^{\prime \prime} A_{m}}{C}\right)^{2}=d \omega \varepsilon_{0} A_{m} \varepsilon^{\prime \prime} \\
& C \omega \varepsilon_{0}{ }^{2} A_{m}{ }^{2} \varepsilon^{\prime 2}+C \omega\left(-\frac{\varepsilon_{0} D \varepsilon^{\prime \prime} A_{m}}{C}\right)^{2}=d \varepsilon_{0} A_{m} \varepsilon^{\prime \prime} \\
& C \omega \varepsilon_{0}{ }^{2} A_{m}{ }^{2} \varepsilon^{\prime \prime}+\frac{\omega \varepsilon_{0}{ }^{2} D^{2} \varepsilon^{\prime \prime} A_{m}^{2}}{C}=d \varepsilon_{0} A_{m} \\
& C^{2} \omega \varepsilon_{0}{ }^{2} A_{m}{ }^{2} \varepsilon^{\prime \prime}+\omega \varepsilon_{0}{ }^{2} D^{2} \varepsilon^{\prime \prime} A_{m}{ }^{2}=C d \varepsilon_{0} A_{m} \\
& \varepsilon^{\prime \prime}\left(C^{2} \omega \varepsilon_{0}{ }^{2} A_{m}{ }^{2}+\omega \varepsilon_{0}{ }^{2} D^{2} A_{m}{ }^{2}\right)=C d \varepsilon_{0} A_{m} \\
& \varepsilon^{\prime \prime}\left(C^{2}+D^{2}\right) \omega \varepsilon_{0}^{2} A_{m}{ }^{2}=C d \varepsilon_{0} A_{m}
\end{aligned}
$$

$$
\begin{gathered}
\varepsilon^{\prime \prime}=\frac{C d}{\left(C^{2}+D^{2}\right) \omega \varepsilon_{0} A_{m}} \\
\varepsilon^{\prime}=-\frac{D \varepsilon}{C}-\frac{B d}{\varepsilon_{0} A_{m}} \\
\varepsilon^{\prime}=-\frac{\frac{D C d}{\left(C^{2}+D^{2}\right) \omega \varepsilon_{0} A_{m}}}{C}-\frac{B d}{\varepsilon_{0} A_{m}} \\
\varepsilon^{\prime}=-\frac{D d}{\left(C^{2}+D^{2}\right) \omega \varepsilon_{0} A_{m}}-\frac{B d}{\varepsilon_{0} A_{m}}
\end{gathered}
$$

Finally

$$
\begin{gathered}
\varepsilon^{\prime}=-\frac{d}{\varepsilon_{0} A_{m}}\left(\frac{D}{\left(C^{2}+D^{2}\right) \omega}+B\right) \\
\varepsilon^{\prime \prime}=\frac{C d}{\left(C^{2}+D^{2}\right) \omega \varepsilon_{0} A_{m}}
\end{gathered}
$$

\subsection{Results with capacitive cell and "liquid type" applicator}

\subsubsection{Measurements in the nominal range of this method}

Measurement have been carried out in $\varnothing_{\text {cell }}=60 \mathrm{~mm}$ cell with cyclohexanol and heptanol.

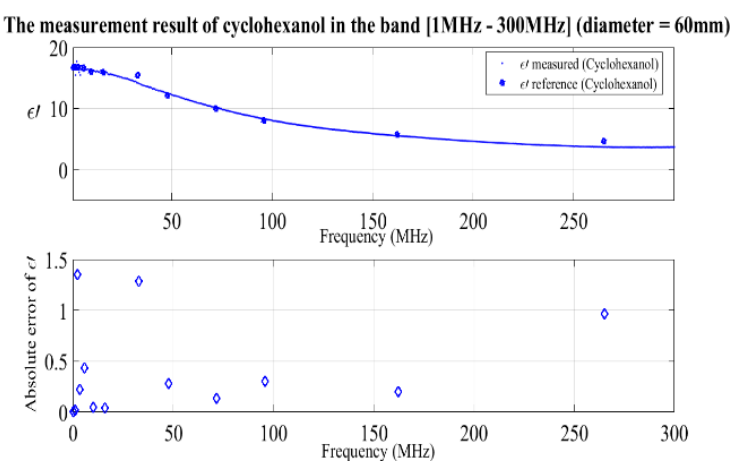

Figure $19 \boldsymbol{\varepsilon}^{\prime}$ - cyclohexanol - [1 MHz - $\left.300 \mathrm{MHz}\right]$

Except below $50 \mathrm{MHz}, \varepsilon^{\prime}$ measured is in good agreement with reference values with an absolute error lower than 1 .

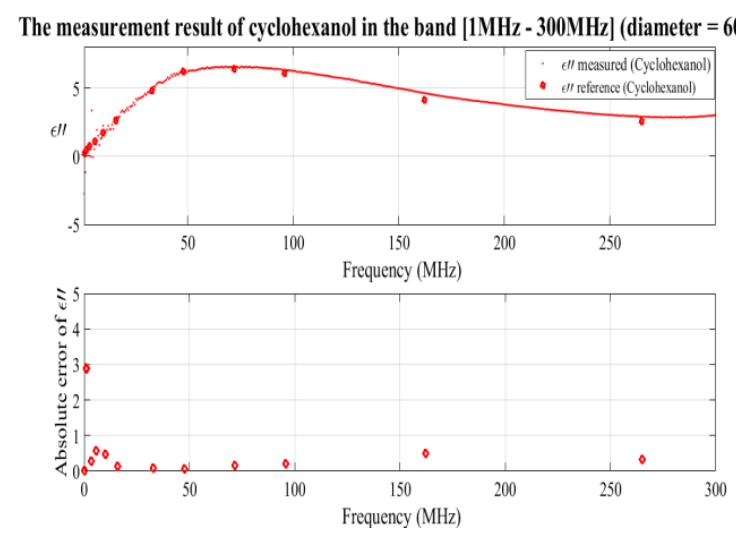

Figure $20 \varepsilon^{\prime \prime}$ - cyclohexanol - [1 MHz - 300 MHz] 
Except below $20 \mathrm{MHz}, \varepsilon^{\prime \prime}$ measured is in good agreement with reference values with an absolute error lower than 1.

The same type of measurement were carried out with the second fluid: heptanol.
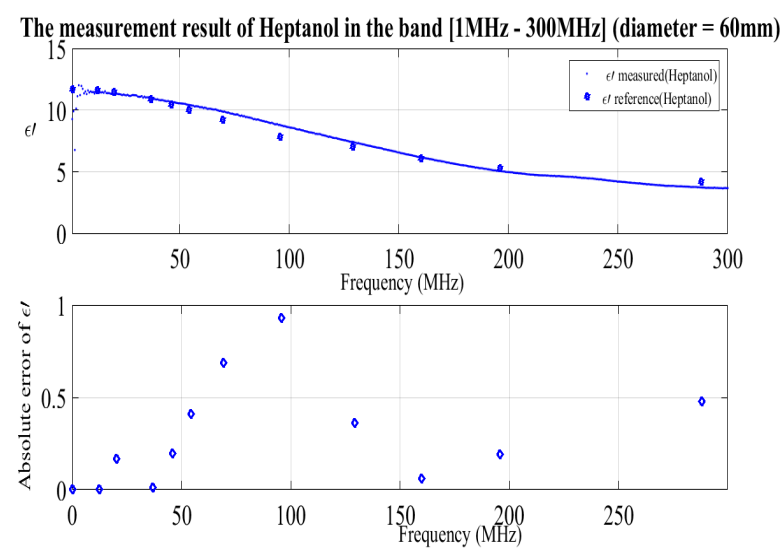

Figure $21 \boldsymbol{\varepsilon}^{\prime}-$ heptanol $-[1 \mathrm{MHz}-300 \mathrm{MHz}]$

The measurement result of Heptanol in the band $[1 \mathrm{MHz}-300 \mathrm{MHz}]($ diameter $=60 \mathrm{~mm})$
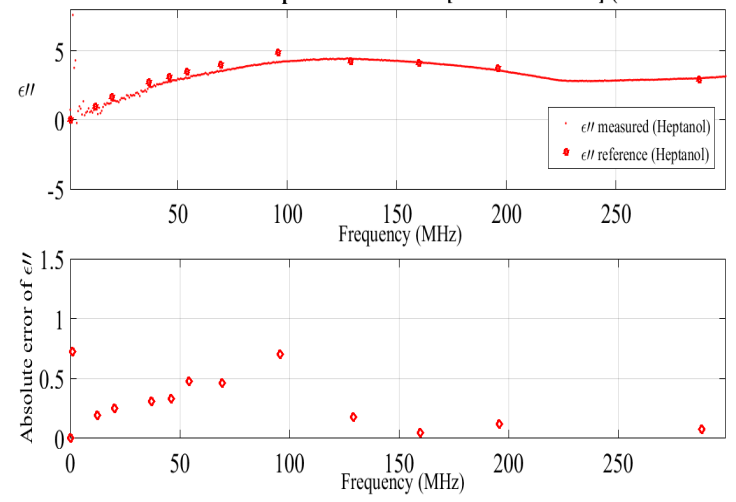

Figure $22 \boldsymbol{\varepsilon}^{\prime \prime}$ - heptanol - [1 MHz - 300 MHz]

The trend noticed with cyclohexanol is confirmed with the second fluid.

\subsubsection{Study of upper and lower limits in frequency}

In order to check the upper limit in terms of frequency, the range is extended up to $500 \mathrm{MHz}$.

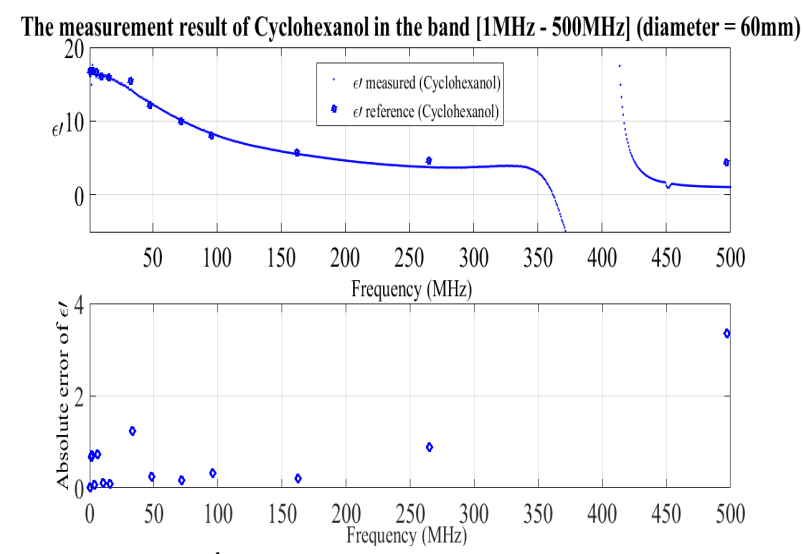

Figure $23 \boldsymbol{\varepsilon}^{\prime}$ - cyclohexanol - [1 MHz - 500 MHz]

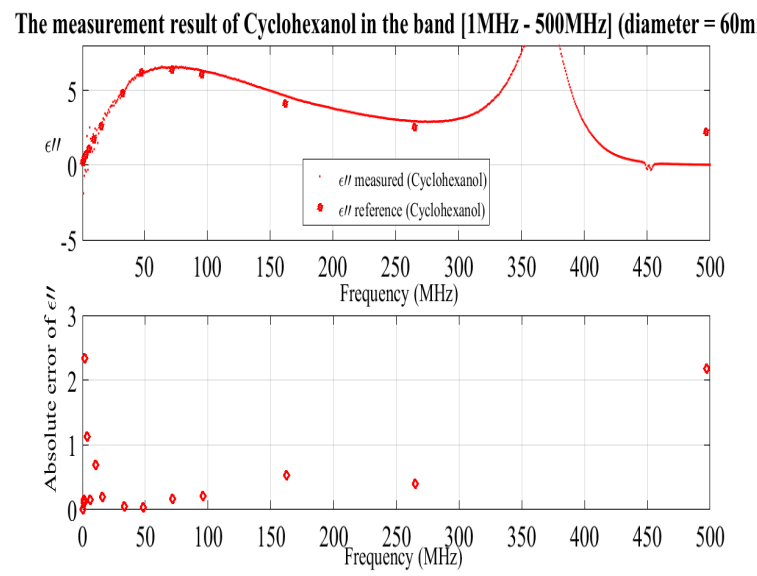

Figure $24 \boldsymbol{\varepsilon}^{\prime \prime}$ - cyclohexanol - [1 MHz - 500 MHz]

In both cases, $\varepsilon^{\prime}$ and $\varepsilon^{\prime \prime}$, a divergence is observed with these dielectric properties. In addition, the absolute error that has been found close to $500 \mathrm{MHz}$ is significantly higher than those obtained below $300 \mathrm{MHz}$.

On the basis of the results obtained, the upper limit of $300 \mathrm{MHz}$ corresponds to the upper limit, in frequency, for this method and measurement device. In the literature, this value is presented to be close to hundreds of $\mathrm{MHz}$ [11].

While measuring in the range $[1 \mathrm{MHz}-300 \mathrm{MHz}$, scattered values has been obtained at low frequency, i.e. below $10 \mathrm{MHz}$. The investigation at frequencies below $10 \mathrm{MHz}$ are presented below.
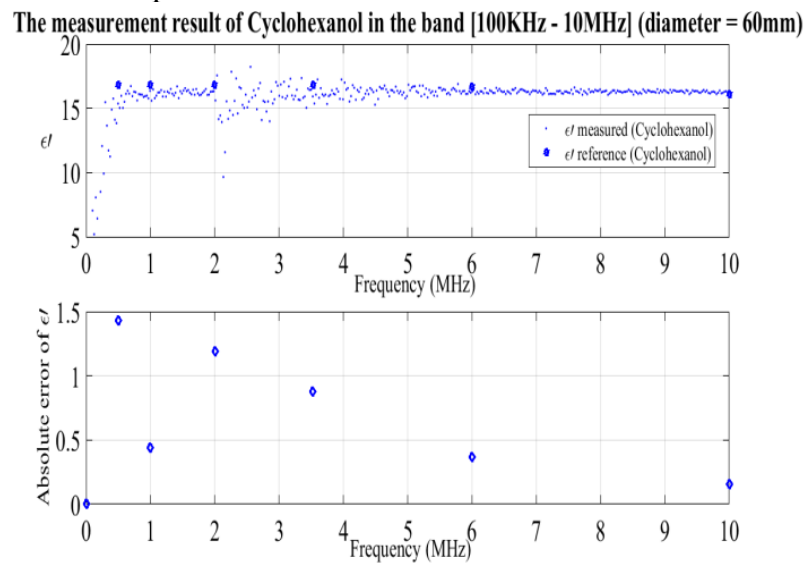

Figure $25 \boldsymbol{\varepsilon}^{\prime}$ - cyclohexanol - [100 kHz - $\left.10 \mathrm{MHz}\right]$

The measurement result of Cyclohexanol in the band $[100 \mathrm{KHz}-10 \mathrm{MHz}]($ diameter $=60 \mathrm{~mm})$
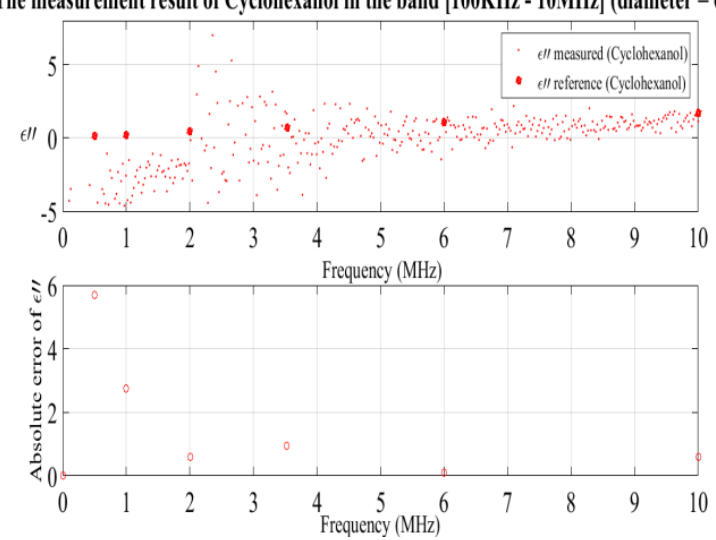

Figure $26 \varepsilon^{\prime \prime}$ - cyclohexanol - [100 kHz - $\left.10 \mathrm{MHz}\right]$ 
Scattered values are more important for $\varepsilon^{\prime \prime}$ than $\varepsilon^{\prime}$, this leads to highest absolute error.

This investigation has been done with the second reference fluid.
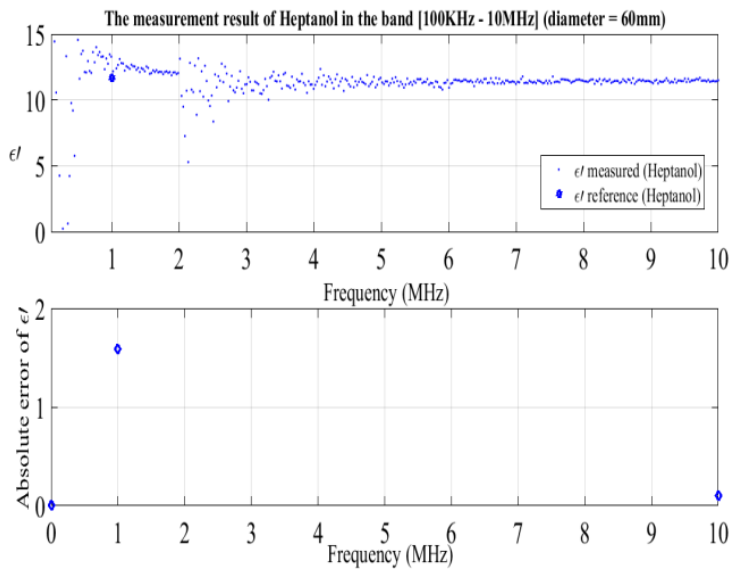

Figure $27 \boldsymbol{\varepsilon}^{\prime}-$ heptanol $-[100 \mathrm{kHz}-10 \mathrm{MHz}]$

The measurement result of Heptanol in the band $[100 \mathrm{KHz}-10 \mathrm{MHz}]($ diameter $=60 \mathrm{~mm}$
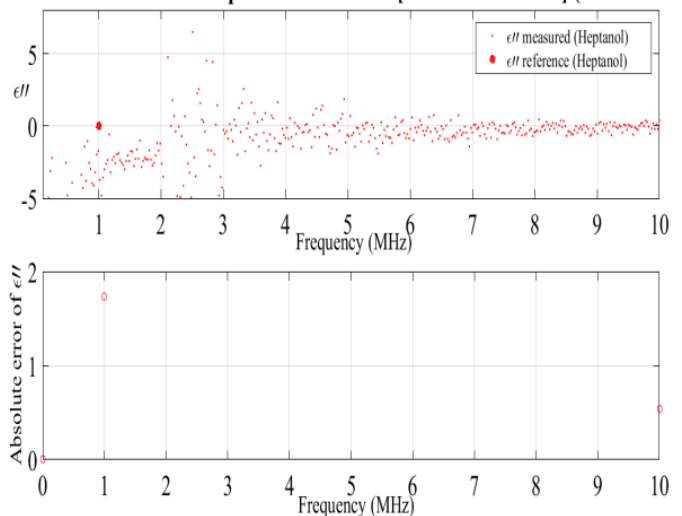

Figure $28 \boldsymbol{\varepsilon}^{\prime \prime}-$ heptanol $-[100 \mathrm{kHz}-10 \mathrm{MHz}]$

The trend is confirmed with the second fluid.

Initially, the scattering phenomenon has not been investigated at low frequencies below $5 \mathrm{MHz}$, but it is a way of optimization of our system which is now limited to $5 \mathrm{MHz}$.

\subsubsection{Study of the inner diameter of the "liquid type" cell}

"Liquid type" cell has been designed with two diameters of quartz ring bounding the fluid. These diameters are lower than the electrodes diameter. This study has been done in order to avoid any edge effects due to the ratio between electrode diameter and diameter of quartz rings.
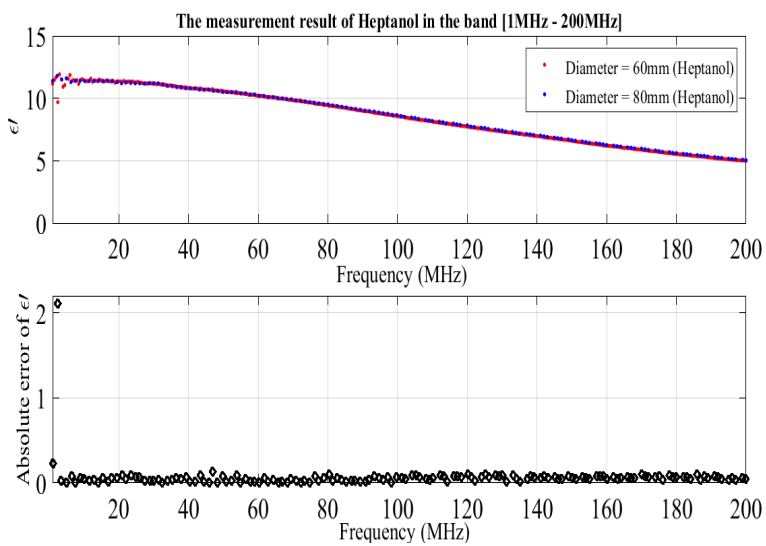

Figure $29 \boldsymbol{\varepsilon}^{\prime}-$ heptanol $-\varnothing 60 \mathrm{~mm}$ and $\varnothing 80 \mathrm{~mm}$ $[1 \mathrm{MHz}-200 \mathrm{MHz}]$
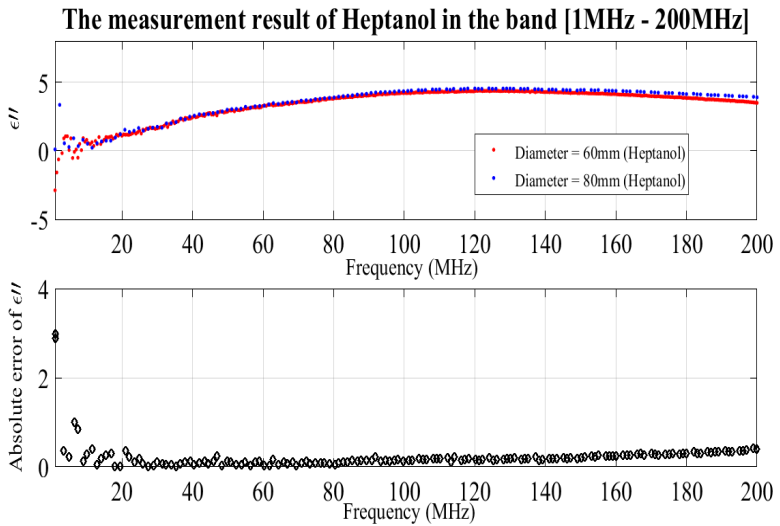

Figure $30 \varepsilon^{\prime \prime}-$ heptanol $-\varnothing 60 \mathrm{~mm}$ and $\varnothing 80 \mathrm{~mm}$ $[1 \mathrm{MHz}-200 \mathrm{MHz}]$

The difference between the results obtained with $60 \mathrm{~mm}$ cell and $80 \mathrm{~mm}$ cell are considered as non significant even though a trend could be seen for the highest frequencies measurement. Thus the cell diameterdoes not have an impact on the measurement when it stays between 60 and $80 \mathrm{~mm}$ in this device.

\section{Conclusion}

This article has presented an original work included in the ERMP project SIB064 METefnet.

The aim of this work is to develop a transfer standard, considered as secondary method requiring calibration against reference method for ensuring SI traceabilty, based on RF and MO principle. As the dielectric permittivity of a material is affected by moisture content, permittivity measurement will lead to moisture measurement.

Two kinds of cells were designed and produced: capacitive cell and coaxial cell. Both can be used to measure solid materials and liquids dielectric propoerties. For the need of characterization, liquids have been selected for studying the capacitive cell.

First results obtained and compared with litterature values with cyclohexanol and heptanol gave confidence in this technics. Upper and lower limits in the frequency range were found for the existing system. We are confident to improve this range in the further steps of this project. 
This project gave also the opportunity for establishing a new collaboration between LNE-CETIAT and Fresnel Institute.

\section{Acknowledgements}

This work was funded by the Joint Research Project SIB64 METefnet of the EMRP, and co-funded through the French metrology LNE-DRST. The EMRP is jointly funded by the EMRP participating countries within EURAMET and the European Union.

\section{References}

1. Heinnonen $M$. et al, " SI traceability to measurements of moisture in solids", Proceedings TEMPMEKO, 2013

2. Martin C., Water Structure and Science, http://www1.1sbu.ac.uk/water/microwave_water. $\underline{\mathrm{html}}$

3. Manoury M., Rochas J.F., Guillemaut H., Chanal B., "Interaction eau-cellulose application au chauffage diélectrique", Revue générale de l'électricité, Vol 11, novembre 1981, p 839 -846

4. Artemov V. G. and Volkov A. A., "Water and ice dielectric spectra scaling at $0{ }^{\circ} \mathrm{C} "$, Ferroelectrics, vol 466, Issue 1, 2014, Special Issue: Proceedings of the Thirteenth International Meeting on Ferroelectricity, DOI: $10.1080 / 00150193.2014 .895216$

5. Encyclopedia of RF and Microwave Engineering, Edited by Kai Chang ISBN 0-471-27053-9 2005 John Wiley \& Sons, Inc.

6. Roussy G., Rochas J.F., Oberlin C., "Chauffage diélectrique - Principes et spécificités", techniques de l'ingénieur D5940, 2008

7. Sabouroux P., Boschi P., "Simplification de la procédure de mesure de caractéristiques électromagnétiques de matériaux en temps réel", REE n8, 2005

8. Sabouroux P., Boschi P., "Epsimu: un nouvel outil pour déterminer les caractéristiques electromagnétiques de matériaux dans le domaine des hyperfréquences", REE, n¹0, 2005

9. Sabouroux P., Ba D., "Epsimu, a tool for dielectric properties measurement of porous media: application in wet granular materials characterizations", Progress In Electromagnetics Research B, Vol. 29, 191-207, 2011

10. Buckley F., Maryott A.. NBS circular 589, 1958

11. Adous M., "Caractérisation électro-magnétique des matériaux traités de génie civil dans la bande de fréquences $50 \mathrm{MHz}$ - $13 \mathrm{GHz}$ ", Thèse de Doctorat de l'Université de Nantes, 2006 